\title{
An idealized wave-ice interaction model without subgrid spatial or temporal discretizations
}

\author{
Luke G. BENNETTS, ${ }^{1}$ Siobhan O'FARRELL, ${ }^{2}$ Petteri UOTILA, ${ }^{2 *}$ Vernon A. SQUIRE ${ }^{3}$ \\ ${ }^{1}$ School of Mathematical Sciences, University of Adelaide, Adelaide, South Australia, Australia \\ E-mail: luke.bennetts@adelaide.edu.au \\ ${ }^{2}$ CSIRO Marine and Atmospheric Research, Aspendale, Victoria, Australia \\ ${ }^{3}$ Department of Mathematics and Statistics, University of Otago, Dunedin, New Zealand
}

\begin{abstract}
A new numerical implementation is proposed for a wave-ice interaction model. It is applied to an idealized transect geometry. Wave attenuation due to ice floes and wave-induced ice fracture are both included in the model. The new method alleviates the need for subgrid spatial or temporal discretizations, thereby facilitating future integration of wave-ice interactions into large-scale coupled models.
\end{abstract}

KEYWORDS: ice/ocean interactions, sea-ice dynamics, sea-ice modelling

\section{INTRODUCTION}

Ocean surface waves have been observed to penetrate tens and, at times, hundreds of kilometres into the sea-icecovered ocean (Kohout and others, 2014). The waves cause the ice to bend and flex. The ice can only endure a certain degree of flexure before it fractures into floes with diameters on the order of local wavelengths (Langhorne and others, 1998). In this way, waves regulate maximum floe sizes. Simultaneously, the presence of ice cover attenuates the waves. Waves therefore retain the intensity to fracture the ice for a finite distance only. Moreover, ice cover filters the wave spectrum preferentially towards low frequencies, so that wavelengths and, consequently, maximum floe sizes generally increase with distance travelled (Squire and Moore, 1980), until winds, currents and further waves reconfigure the simple arrangement.

Wave attenuation is typically modelled as an accumulation of partial wave reflections and transmissions by floes (see Bennetts and Squire, 2012, and references therein). Comparatively few fracture models exist. Those that do exist are based on wave-induced flexural motion of the ice, resulting in strains that exceed a threshold (e.g. Langhorne and others, 2001).

Until recently, wave attenuation and ice fracture models have been independent of one another. Attenuation models consider the floe size distribution to be known. Fracture models consider the distribution of wave energy to be known. However, wave attenuation and ice fracture are coupled via the floe size distribution. The rate of wave attenuation depends on the floe size distribution, which is regulated by wave-induced fracture. Fracture depends on the local wave energy, which is controlled by wave attenuation imposed by the ice cover (Kohout and Meylan, 2008).

Dumont and others (2011) and Williams and others (2013a,b) developed a wave-ice interaction model (WIM), in which wave attenuation and ice fracture are coupled processes. The WIM predicts the distribution of wave energy and the resulting floe size distribution simultaneously, given an incident wave forcing from the open ocean and properties of the ice cover.

*Present address: Finnish Meteorological Institute, Helsinki, Finland.
Dumont and others (2011) and Williams and others $(2013 a, b)$ designed the WIM to link wave and sea-ice model components of large-scale operational ice-ocean forecasting models. The WIM is nested in regions of operational interest in the large-scale models using refined spatial and temporal grids. The WIM then provides prognostic information regarding the floe size distribution and wave activity in the ice-covered ocean, potentially leading to more accurate forecasts for the safety of offshore engineering activities.

More generally, the WIM provides an opportunity to integrate prognostic wave and floe size information into oceanic general circulation models (OGCMs) used for climate studies, and here considered to contain wave and sea-ice model components. The information will improve the accuracy of existing models of floe-size-dependent processes in the vicinity of the ice edge, such as form drag (Tsamados and others, 2014), and promote development of new models of wave- and floe-size-dependent processes, such as accelerated melt of floes due to wave overwash (Massom and Stammerjohn, 2010).

Subgridding incurs a large computational cost. It is therefore not an appropriate method to implement the WIM on circumpolar or global scales. An alternative numerical implementation is proposed here, for a modified version of the WIM. The new method does not involve subgrid spatial or temporal discretizations. Instead, the floe size distribution and the rate of attenuation are balanced in the cell.

Application of the new scheme to an idealized version of the modified WIM is presented. Results are validated by comparison to results produced by the method of Williams and others (2013b).

\section{MODEL DESCRIPTION}

Consider a transect of the ocean surface containing ice cover. Points on the transect are defined by the coordinate $x \in(0, L)$. The transect represents a single cell in an OGCM. The time frame under consideration, $t \in(0, T)$, is equivalent to a single time step in the OGCM. For climate models $L \sim 50 \mathrm{~km}$ and $T \sim 1$ hour. The sea-ice model component of the OGCM is assumed to provide an average ice thickness, $h$, and concentration of the ice cover, c. (Contemporary 
sea-ice models distribute ice thickness over multiple categories.) The average ice thickness $h=0.5 \mathrm{~m}$ and concentration $c=0.75$ are prescribed for the idealized model results presented later.

Let the wave energy density spectrum at point $x$ and time $t$ be denoted $S(\omega ; x, t)$, where $\omega$ is angular frequency. The domain $x \in(0, L)$ is considered to be free of waves initially. An incident wave spectrum is prescribed at the left-hand end of the domain, $x=0$. Incident wave forcing is steady over the time interval considered.

The incident spectrum is transported through the transect according to the energy-balance equation

$$
\frac{1}{c_{\mathrm{g}}} \frac{\partial S}{\partial t}+\frac{\partial S}{\partial x}=-\alpha S
$$

The quantity $c_{\mathrm{g}}$ is the group velocity of the waves. Williams and others (2013b) provide numerical evidence that ice fracture predicted by the WIM is insensitive to wave dispersion. Although the finding is not conclusive, for simplicity dispersion is neglected for the present application, i.e. a constant value of $c_{\mathrm{g}}$ is used in the transport equation. Further, it is assumed that the wave spectrum travels the length of the transect in the time frame, i.e. $C_{\mathrm{g}}=L / T$, thus alleviating the need to specify a value for $c_{\mathrm{g}}$.

The quantity $\alpha(\omega ; x, t)$ is the attenuation coefficient, i.e. the exponential attenuation rate of wave energy per meter. The expression $\alpha=c a /\langle d\rangle$ is used here, where $d$ denotes floe diameter, $\langle\cdot\rangle$ denotes the mean of the included quantity and $a(\omega, h)$ is the attenuation rate per floe, calculated using the model of Bennetts and Squire (2012). The attenuation coefficient therefore depends on ice thickness and concentration. It also depends on the floe size distribution, which is calculated by the WIM. The floe size distribution is defined by its probability density function $p(d ; x, t)$. The floe size distribution, and hence the attenuation coefficient, vary suddenly in time if wave-induced ice fracture occurs. The occurrence of fracture, in turn, depends on the wave energy spectrum. Calculation of the wave energy spectrum, $S$, and the floe size distribution, $p$, are therefore coupled via the attenuation coefficient.

In order to isolate wave-ice interactions, the above version of the transport equation (1) neglects nonlinear transfer of wave energy between frequencies, wave energy input due to winds, long-range attenuation of swell, and attenuation due to wave breaking and whitecapping. To the authors' knowledge, the importance of these processes in the ice-covered ocean is not yet known. The fracture model derived by Williams and others (2013a) is used. The model is based on strains imposed on the ice cover by wave motion, in which the ice is modelled as a thin-elastic beam. The fracture condition is

$$
E \geq E_{\mathrm{C}}=\varepsilon_{\mathrm{C}} \sqrt{-2 / \log \left(\mathrm{P}_{\mathrm{C}}\right)},
$$

where $E=2 \sqrt{m_{0}[\varepsilon]}$ is the strain imposed on the ice by the waves. The quantity $m_{0}$ is the zeroth moment of strain. The $n$th moment of strain is defined as

$$
m_{n}[\varepsilon]=\frac{h^{2}}{4} \int_{0}^{\infty} \omega^{n} \kappa^{4}(\omega) S(\omega) \mathrm{d} \omega,
$$

where $\kappa$ is the ice-coupled wavenumber (Williams and others, 2013a). The quantity $\varepsilon_{C}=\sigma_{C} / Y^{*}$ is the fracture limit of the ice, where $\sigma_{C}$ is the flexural strength of the ice, $Y^{*}$ is the effective Young's modulus of the ice and $\mathrm{P}_{\mathrm{C}}$ is a chosen critical probability.

The expression $\sigma_{c}=1.76 \exp \left(-5.88 \sqrt{\nu_{\mathrm{b}}}\right) \mathrm{MPa}$ is used for the flexural strength, in which $\nu_{\mathrm{b}}$ denotes brine volume fraction. Timco and O'Brien (1994) used 939 flexural strength measurements to derive the expression. (The root brine volume fraction ranged from 0.15 to 0.5 , approximately, for those measurements obtained in situ using cantilever beams.)

The expression $Y^{*}=\left\{10\left(1-3.51 \nu_{\mathrm{b}}\right)-1\right\} \mathrm{GPa}$, as derived by Williams and others (2013a), is used for the effective Young's modulus. (Williams and others noted that in situ measurements on large samples of sea ice often produce smaller values of the effective Young's modulus.)

The critical probability limit for a narrow-band spectrum, $\mathbb{P}_{\mathrm{c}}=\exp (-1)$, is used. Williams and others (2013b) conducted a numerical study of the sensitivity of ice fracture to the value of the critical probability.

Fracture occurs if inequality (2) is satisfied. If fracture occurs, the maximum floe diameter is set to be equal to half of the dominant wavelength, i.e. $d_{\mathrm{mx}}=\lambda / 2$. The dominant wavelength is calculated from the dispersion relation for the ice-covered ocean (e.g. Bennetts and Squire, 2011), using the dominant wave period

$$
\tau=2 \pi \sqrt{\frac{m_{0}[\eta]}{m_{2}[\eta]}}, \quad \text { where } m_{n}[\eta]=\int_{0}^{\infty} \omega^{n} S(\omega) \mathrm{d} \omega
$$

is the $n$th spectral moment of the surface elevation $\eta(\mathrm{WMO}$, 1998). The maximum floe diameter parameterizes the floe size distribution. The floe size distribution is otherwise specified. A truncated power-law floe size distribution is used. The corresponding probability density function is

$$
p(d)=\left\{\begin{array}{cc}
\frac{\gamma d_{\mathrm{mn}}^{\gamma} d^{-(\gamma+1)}}{1-\left(d_{\mathrm{mn}} / d_{\mathrm{mx}}\right)^{\gamma}} & \text { if } d \in\left[d_{\mathrm{mn}}, d_{\mathrm{mx}}\right], \\
0 & \text { if } d \notin\left[d_{\mathrm{mn}}, d_{\mathrm{mx}}\right],
\end{array}\right.
$$

where $d_{\mathrm{mn}}=20 \mathrm{~m}$ is a chosen minimum floe diameter and $\gamma=2+\log _{2}(0.9)$ is the chosen exponent (Dumont and others, 2011; Toyota and others, 2011; Williams and others, 2013a).

Williams and others (2013b) used numerical results to show that WIM ice-fracture predictions are insensitive to the proportion of wave energy lost to ice fracture, if $>30 \%$ of wave energy is lost to fracture. Experimental data do not exist to support or refute the assumption. It is therefore assumed that all wave energy is lost, i.e. steady-state conditions apply.

\section{NUMERICAL IMPLEMENTATION Method of Williams and others (2013b)}

Williams and others (2013b) applied spatial, temporal and spectral discretizations to the WIM. For each time step the following sequence of executions is applied:

1. Advection: The discretized wave spectrum is mapped to an intermediate spectrum by solving the energy-balance equation (1) without attenuation, i.e. $\alpha=0$.

2. Attenuation: The intermediate wave spectrum is attenuated according to the properties of the ice it has just travelled through.

3. Fracture: In each cell, fracture condition (2) is applied. If the wave spectrum in a given cell is sufficient to cause fracture, the maximum floe diameter in the cell is set to 

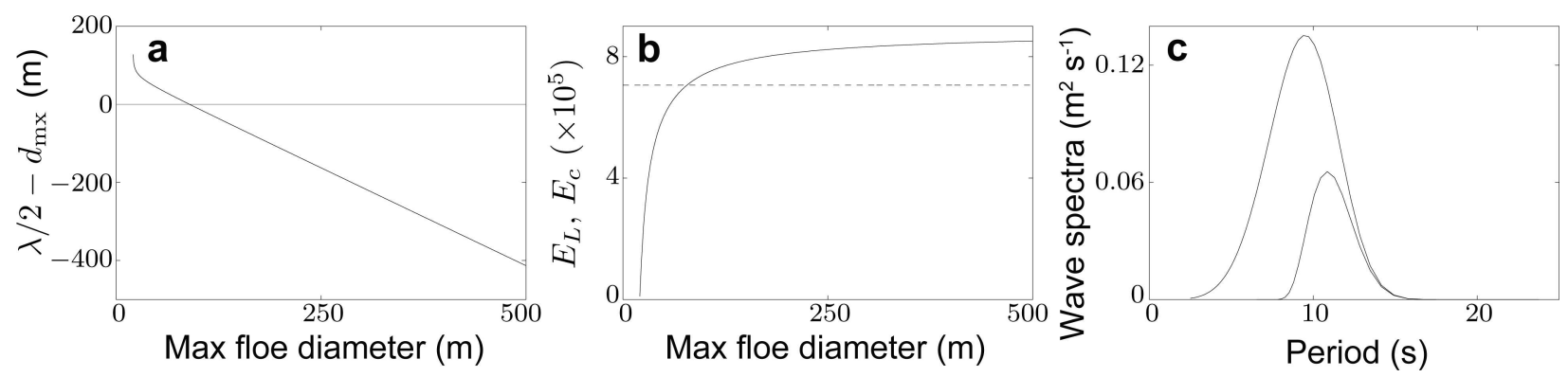

Fig. 1. Results for incident wave spectrum with $1 \mathrm{~m}$ significant wave height and $9.5 \mathrm{~s}$ peak period, and transect length $L=100 \mathrm{~km}$. (a) Black curve represents half-wavelength/maximum-floe-diameter balance function at $x=L$. Grey curve represents abscissa axis. (b) Solid curve represents strain imposed on the ice by wave spectrum at $x=L$, as a function of maximum floe diameter. Broken curve represents the strain threshold $E_{c}$. (c) Incident wave spectrum and attenuated wave spectrum at $x=L$.

half of the dominant wavelength. However, if the dominant wavelength is greater than the existing maximum diameter, no change is made.

Williams and others $(2013 \mathrm{~b})$ set the initial maximum diameters to a large value $\sim 500 \mathrm{~m}$, in cells where the maximum floe diameter is not otherwise initialized.

\section{New method without spatial or temporal discretizations}

An ice cover acts as a low-pass filter to waves, i.e. the attenuation rate decreases as frequency decreases/period increases. Consequently, the wave spectrum increasingly skews towards low frequencies the farther it propagates into the ice-covered ocean, and the associated wavelength becomes larger. The maximum floe diameter thus increases with distance away from the ice edge in the wave-induced fracture region.

The low-pass filter observation is the basis of an alternative numerical implementation of the WIM proposed below, which does not require spatial or temporal discretizations. The observation is translated to the assumption that the maximum floe diameter is due to ice fracture at the far end of the transect, $x=L$, or the farthest distance into the transect for which the wave spectrum is capable of causing fracture.

The problem reduces to (1) determining if the wave spectrum at $x=L$ is sufficient to cause ice fracture, and, if not, (2) determining the farthest point into the transect at which the wave spectrum is capable of causing ice fracture. However, the wave spectrum and the maximum floe diameter must be calculated simultaneously, because they are coupled via the attenuation coefficient.

Suppose a trial value is specified for the maximum floe diameter, and hence the attenuation coefficient is known. The wave spectrum at $x=L$ is then obtained directly from the energy-balance equation (1). The associated strain, $E_{L}\left(d_{\mathrm{mx}}\right)$, and wavelength, $\lambda_{L}\left(d_{\mathrm{mx}}\right)$, at $x=L$ are calculated as described at the end of Section 2. Three scenarios are possible:

1. the maximum floe diameter is less than half the wavelength;

2. the maximum floe diameter is greater than half the wavelength; and

3. the maximum floe diameter is equal to half the wavelength.
Scenario 3 is sought here. In scenario 3 the wavelength and the maximum floe diameter balance one another, i.e. the maximum diameter defines a floe size distribution that attenuates the wave spectrum such that the wavelength at $x=L$ is consistent with the maximum diameter. The initial floe diameter is considered to be large. Note that the new method does not require a specific value to be prescribed for the initial maximum diameter.

The maximum diameter that balances the wavelength at $x=L$ is obtained by finding the value of $d_{\mathrm{mx}}$ that satisfies the equation

$$
\frac{1}{2} \lambda_{L}\left(d_{\mathrm{mx}}\right)=d_{\mathrm{mx}}
$$

However, for this value of $d_{m x}$, fracture only occurs if the strain imposed by the wave spectrum on the ice cover at $x=L$ satisfies the inequality

$$
E_{L}\left(d_{\mathrm{mx}}\right) \geq E_{c} .
$$

Equation (6) can be solved numerically using a package root-finding algorithm (e.g. MATLAB ${ }^{\mathrm{TM}^{\prime}}$ 's f zero function). A check of fracture condition (7) is then performed for the calculated value of $d_{\mathrm{mx}}$.

Figure 1 shows results for a problem in which fracture occurs at $x=L=100 \mathrm{~km}$. A Bretschneider incident wave spectrum is used. It is defined by a peak period and a significant wave height, which is convenient for model testing. For this problem, the incident peak period is $9.5 \mathrm{~s}$ and the significant wave height is $1 \mathrm{~m}$. However, any incident wave spectrum can be used, including ones derived from observations or wave models such as WAM or Wavewatch.

Figure 1a shows the difference between the halfwavelength at $x=L$ and the maximum floe diameter, for a given maximum floe diameter. The half-wavelength and maximum floe diameter balance one another at $\sim 89.5 \mathrm{~m}$. Figure $1 \mathrm{~b}$ shows the corresponding strain imposed by the wave spectrum on the ice cover, as a function of maximum floe diameter, and the strain threshold, $E_{c}$. It confirms that the wave spectrum is sufficiently intense to fracture the ice cover when $d_{\mathrm{mx}} \approx 89.5 \mathrm{~m}$.

The maximum floe diameter is therefore set as $d_{\mathrm{mx}} \approx 89.5 \mathrm{~m}$. Figure $5 \mathrm{c}$ shows the incident wave spectrum and the attenuated wave spectrum at $x=L$. Skew of the attenuated spectrum towards long periods is clear.

If the fracture condition is not satisfied at $x=L$, the problem is extended to search for the greatest distance into the transect at which the wave spectrum is capable of causing fracture. The extension is necessary, as wave-induced 

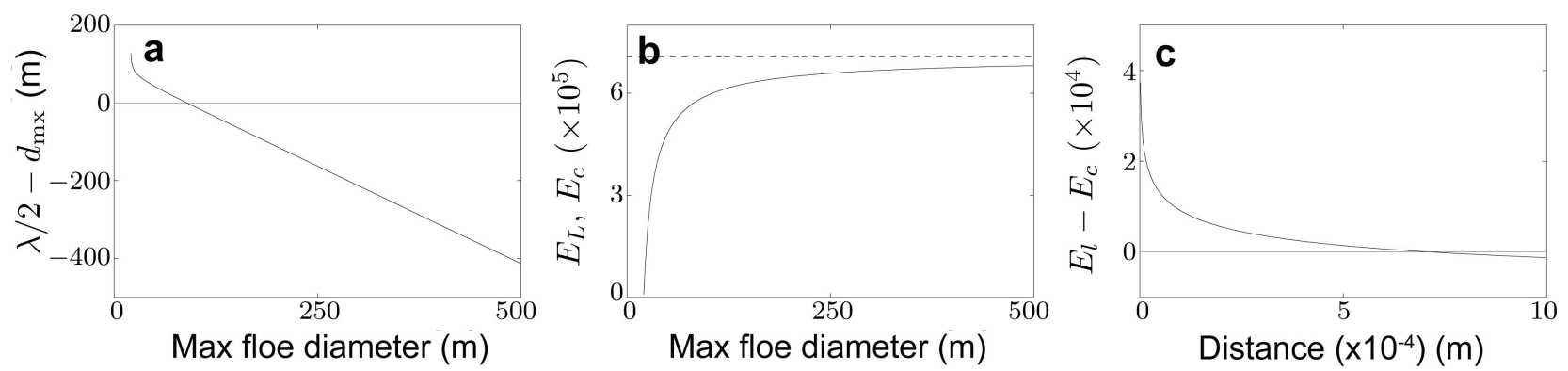

Fig. 2. (a, b) Same as Figure 1 but for $0.8 \mathrm{~m}$ incident significant wave height. (c) Corresponding strain balance equation as a function of distance, I. In (b) and (c) grey curves represent abscissa axes.

fracture may be possible for a large proportion of the transect, despite not being possible for the entire transect.

Let $x=/$ be the greatest distance at which fracture can occur. The strain imposed on the ice cover by the wave spectrum at this point, $E_{l}\left(d_{\mathrm{mx}}\right)$, is such that

$$
E_{l}\left(d_{\mathrm{mx}}\right)=E_{\mathrm{c}} \text {. }
$$

Additionally, the wavelength at $x=I, \lambda_{l}$, must balance the maximum diameter, i.e.

$$
\frac{1}{2} \lambda_{l}\left(d_{\mathrm{mx}}\right)=d_{\mathrm{mx}}
$$

Equations (8a) and (8b) are to be solved simultaneously for / and $d_{\mathrm{mx}}$. In practice, the equations are solved numerically as a set of one-dimensional equations:

1. by solving Eqn (8b) to give the maximum diameter that balances the wave spectrum, as a function of distance $I$, i.e. $d_{\mathrm{mx}}=d_{\mathrm{mx}}(I)$; and

2. substituting the expression for $d_{\mathrm{mx}}=d_{\mathrm{mx}}(I)$ into Eqn (8a) and solving for the distance $l$.

The extended method is necessary for a problem identical to that used above (Fig. 1) but with a $0.8 \mathrm{~m}$ significant wave height for the incident spectrum. Figure 2 shows results produced by the extended method. As in Figure 1, the lefthand and middle panels show the balance equation and strains at $x=L$. The half-wavelength and maximum floe diameter again balance one another at $\sim 89.5 \mathrm{~m}$. However, the wave spectrum at $x=L=100 \mathrm{~km}$ is not sufficiently intense to fracture the ice for $d_{\mathrm{mx}} \approx 89.5 \mathrm{~m}$ (or for any $d_{\mathrm{mx}}$ less than 500 m; Fig. 2b). Figure 2c shows the strain balance equation as a function of distance $I$. The largest distance at which the wave spectrum remains sufficiently intense to fracture the ice is $\sim 71.3 \mathrm{~km}$, i.e. wave-induced fracture occurs over a substantial proportion of the transect.

The two numerical implementations use different interpretations of the floe size distribution. The new method uses a single maximum floe diameter for the transect, which, recall, represents a single cell in an OGCM. The floe size distribution, $p$, for the entire transect is parameterized by the maximum floe diameter. In contrast, the method of Williams and others (2013b) considers the floe size distribution to be a property of the subcells of the transect, i.e. a maximum floe diameter is assigned to each subcell. The floe size distribution for the entire transect is generally not of the form $p$.

Figure 3 compares results produced by the two methods, for an example problem. Figure 3a shows fracture width, defined as the distance of the transect over which waveinduced fracture occurs, as a function of the peak period of the incident wave spectrum. Figure $3 \mathrm{~b}$ shows maximum floe diameter in the fractured region, as a function of peak period. The length of the transect is set to be very large so that the fractured region is not truncated. The significant wave height of the incident spectrum is $1 \mathrm{~m}$.

Fracture width and maximum floe diameter both increase as peak period increases. The increase in fracture width becomes less rapid as peak period increases. The relationship between maximum floe diameter and peak period is approximately linear for the interval considered.

Given the different interpretations of the floe size distribution in the two methods, the agreement in the results is reassuring. (Slight differences for the predicted fracture
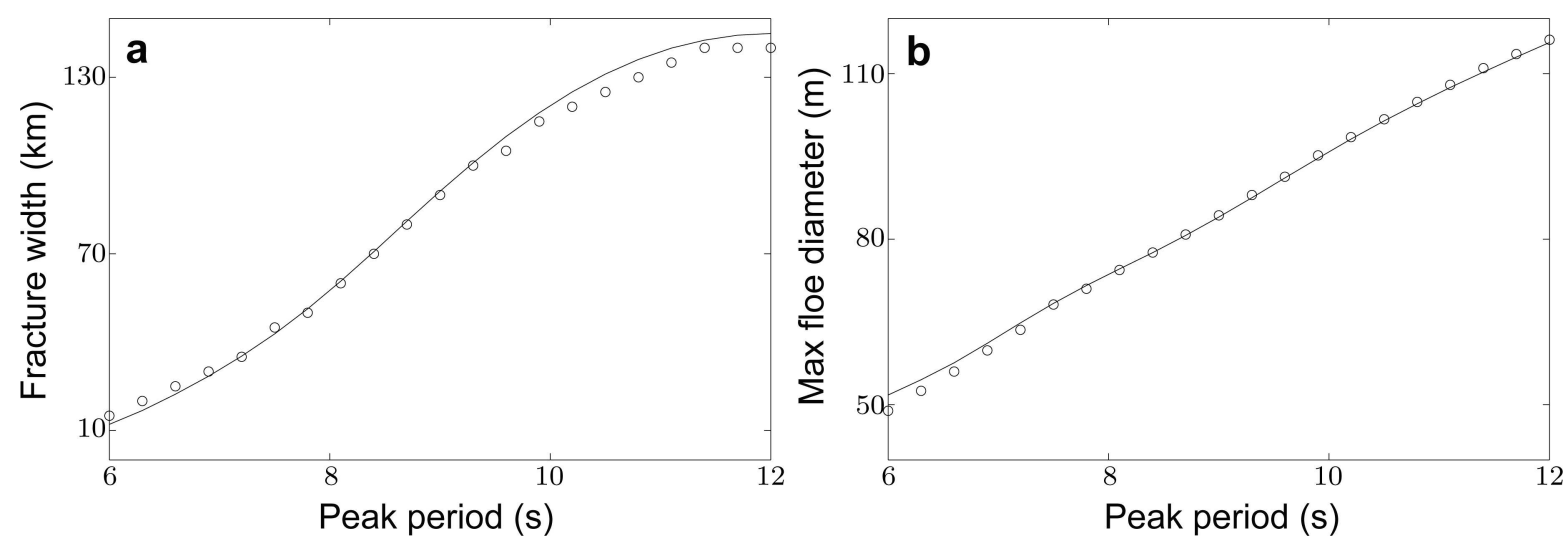

Fig. 3. Fracture width (a) and maximum floe diameter (b), as functions of incident wave spectrum peak period. Incident wave spectrum significant wave height is $1 \mathrm{~m}$. Circles and curves denote results produced by method of Williams and others (2013b) and new method, respectively. 
widths can be attributed, in part, to discontinuities resulting from discretization of the transect in the method of Williams and others $(2013 \mathrm{~b})$ - a $5 \mathrm{~km}$ cell length was used for the example.) The results therefore indicate the WIM is insensitive to precise knowledge of the floe size distribution.

\section{SUMMARY AND DISCUSSION}

A new numerical implementation has been applied to a modified version of the WIM proposed by Williams and others (2013b). The new method was based on the assumption that the maximum floe diameter occurs at the far end of the cell. At the far boundary the dominant wavelength is at its maximum, as the wave attenuation due to ice cover skews the wave spectrum towards long period waves.

The maximum floe diameter was used to define the floe size distribution in the cell. A wavelength/maximum-floesize equation was posed on the far boundary and solved to obtain the wave attenuation rate and floe size distribution, simultaneously. If the wave spectrum was not sufficiently intense to fracture the ice at the far boundary, the problem was extended to search for the farthest distance into the cell at which fracture occurs.

The new method was presented for an idealized transect geometry. Example results for the width of the fractured ice region, and maximum floe diameter due to wave-induced fracture, as functions of peak wave period were given. The results were compared to results produced by the method of Williams and others (2013b). The two methods gave almost identical results. Close agreement was not guaranteed, as the methods adopt different interpretations of the floe size distribution.

Integration of the WIM into OGCMs will require, in the first instance, an extension to two-dimensional (2-D) geometries. The key assumption of the new method, which is that the maximum floe diameter occurs at the far end of a cell, can be reinterpreted directly in the 2-D setting. However, the validity of the key assumption must be tested for directional wave spectra and multiple thickness categories.

\section{ACKNOWLEDGEMENTS}

The Australian Research Council (DE130101571) and Australian Antarctic Science Program (Project 4123) fund L.G.B. The Office of Naval Research (N00014-131-0279) funds V.A.S.

\section{REFERENCES}

Bennetts LG and Squire VA (2012) On the calculation of an attenuation coefficient for transects of ice-covered ocean. Proc. R. Soc. London, Ser. A, 468(2137), 136-162 (doi: 10.1098/ rspa.2011.0155)

Dumont D, Kohout A and Bertino L (2011) A wave-based model for the marginal ice zone including a floe breaking parameterization. J. Geophys. Res., 116(C4), C04001 (doi: 10.1029/ 2010JC006682)

Kohout AL and Meylan MH (2008) An elastic plate model for wave attenuation and ice floe breaking in the marginal ice zone. J. Geophys. Res., 113(C9), C09016 (doi: 10.1029/ 2007JC004434)

Kohout AL, Williams MJM, Dean SM and Meylan MH (2014) Storminduced sea-ice breakup and the implications for ice extent. Nature, 509(7502), 604-607 (doi: 10.1038/nature13262)

Langhorne PJ, Squire VA, Fox C and Haskell TG (1998) Break-up of sea ice by ocean waves. Ann. Glaciol., 27, 438-442

Langhorne PJ, Squire VA, Fox C and Haskell TG (2001) Lifetime estimation for a land-fast ice sheet subjected to ocean swell. Ann. Glaciol., 33, 333-338 (doi: 10.3189/172756401781818419)

Massom RA and Stammerjohn SE (2010) Antarctic sea ice change and variability - physical and ecological implications. Polar Sci., 4(2), 149-186 (doi: 10.1016/j.polar.2010.05.001)

Squire VA and Moore SC (1980) Direct measurement of the attenuation of ocean waves by pack ice. Nature, 283(5745), 365-368 (doi: 10.1038/283365a0)

Timco GW and O'Brien S (1994) Flexural strength equation for sea ice. Cold Reg. Sci. Technol., 22(3), 285-298 (doi: 10.1016/ 0165-232X(94)90006-X)

Toyota T, Haas C and Tamura T (2011) Size distribution and shape properties of relatively small sea-ice floes in the Antarctic marginal ice zone in late winter. Deep-Sea Res. II, 58(9-10), 1182-1193 (doi: 10.1016/j.dsr2.2010.10.034)

Tsamados M, Feltham DL, Schroeder D and Flocco D (2014) Impact of variable atmospheric and oceanic form drag on simulations of Arctic sea ice. J. Phys. Oceanogr., 44(5), 1329-1353 (doi: 10.1175/JPO-D-13-0215.1)

Williams TD, Bennetts LG, Squire VA, Dumont D and Bertino L (2013a) Wave-ice interactions in the marginal ice zone. Part 1: Theoretical foundations. Ocean Model., 71, 81-91 (doi: 10.1016/j.ocemod.2013.05.010)

Williams TD, Bennetts LG, Squire VA, Dumont D and Bertino L (2013b) Wave-ice interactions in the marginal ice zone. Part 2: Numerical implementation and sensitivity studies along 1D transects of the ocean surface. Ocean Model., 7, 92-101 (doi: 10.1016/j.ocemod.2013.05.011)

World Meteorological Organization (WMO) (1998) Guide to wave analysis and forecasting, 2nd edn. (WMO-No 702) World Meteorological Organization, Geneva 